\title{
Travelling fronts in stochastic Stokes' drifts
}

\author{
Adrien Blanchet ${ }^{\mathrm{a}}$ \\ ${ }^{a} D A M T P$, Centre for Mathematical Sciences, Wilberforce Road, Cambridge CB3 \\ oWA, United Kingdom. \\ Jean Dolbeault ${ }^{b}$ \\ ${ }^{\mathrm{b}}$ Ceremade (UMR CNRS no. 7534), Université Paris-Dauphine, Place de Lattre \\ de Tassigny, 75775 Paris Cedex 16, France. \\ Michał Kowalczyk ${ }^{\mathrm{c}}$ \\ ${ }^{\mathrm{c}}$ Universidad de Chile, Facultad de Ciencias Físicas y Matemáticas, Depto \\ Ingeniería Matemática, Blanco Encalada 2120, Piso 5, Santiago, Chile.
}

\begin{abstract}
By analytical methods we study the large time properties of the solution of a simple one-dimensional model of stochastic Stokes' drift. Semi-explicit formulae allow us to characterize the behaviour of the solutions and compute global quantities such as the asymptotic speed of the center of mass or the effective diffusion coefficient. Using an equivalent tilted ratchet model, we observe that the speed of the center of mass converges exponentially to its limiting value. A diffuse, oscillating front attached to the center of mass appears. The description of the front is given using an asymptotic expansion. The asymptotic solution attracts all solutions at an algebraic rate which is determined by the effective diffusion coefficient. The proof relies on an entropy estimate based on homogenized logarithmic Sobolev inequalities. In the traveling frame, the macroscopic profile obeys to an isotropic diffusion. Compared with the original diffusion, diffusion is enhanced or reduced, depending on the regime. At least in the limit cases, the rate of convergence to the effective profile is always decreased. All these considerations allow us to define a notion of efficiency for coherent transport, characterized by a dimensionless number, which is illustrated on two simple examples of traveling potentials with a sinusoidal shape in the first case, and a sawtooth shape in the second case.
\end{abstract}

Key words: Stochastic Stokes' drift, drift velocity, effective diffusion, ratchet, Brownian motion, molecular motors, transport coherence PACS: 05.40.-a, 02.30.Jr, 05.40.Jc, 87.10.Ca, 87.10.Ed, 87.15.hj, 87.15.Vv 


\section{Introduction}

The stochastic Stokes' drift, see [1], is a simple model describing the diffusion of particles in the presence of a periodic, wave-like potential. Particles suspended in a liquid and subject to diffusion experience a net drift due to the wave traveling through the liquid. It can also be seen as a simple model of Brownian ratchet. When there is no diffusion, the net drift of particles is proportional to $\omega$ when $\omega$ is small, but decays to 0 when $\omega$ is large. In the presence of a diffusion the situation is different since, due to the Brownian motion, some particles will move in the direction opposite to the wave train. Our goal is to study the net drift, or to be precise, the speed of the center of mass, the formation of the front and its diffusion, when there are no spatial limitation for the solution, and to measure the efficiency in terms of coherent transport.

From the mathematical point of view, we shall primarily refer to [2] and references therein. In [2] we studied the stochastic Stokes' drift from a theoretical point of view. This paper is intended to give a more descriptive approach of the diffusive front by methods of partial differential equations. Questions related to the stochastic Stokes' drift have been studied before in the context of diffusive turbulent flows, see $[3,4]$. Also see [5] for drift velocity related issues. The effective diffusion coefficient is determined by the traveling potential. Similar effects are known in the context of homogenization theory, see, e.g., $[5,6]$. The stochastic Stokes' drift has the additional difficulty that it is an evolution problem in which the small parameter and the time are not independent. We will provide some asymptotic expansions which are also inspired by the homogenization theory, and put some emphasis on rates of convergence, which are a major pending question from a numerical point of view.

The literature on the stochastic Stokes' drift and Brownian ratchets is huge. We first refer to [7]: the drift velocity is computed in the case of a sinusoidal traveling potential (also see $[8,9]$ ) and the diffuse traveling front is exhibited on the basis of numerical results. Brownian ratchets generically refer to drift-diffusion models in which a time periodic forcing coupled to some asymmetry induces a transport at large scale. The notion of traveling potential is explored in $[10,11]$. We refer to [12] and references therein for the notion of tilted Smoluchowski-Feynman ratchet, which makes an explicit connection between the stochastic Stokes' drift and ratchet mechanisms. As we shall see later, a change of variables indeed reduces the model to a simple tilted Brownian ratchet, with no more explicit time-dependence. An historical perspective of the physics of ratchets and useful definitions are given in [11,13]. Many important issues, like effects due to the asymmetry of the potential, the geometry

Email addresses: blanchet@ceremade.dauphine.fr (Adrien Blanchet), dolbeaul@ceremade.dauphine.fr (Jean Dolbeault), kowalczy@dim.uchile.cl (Michał Kowalczyk).

URLs: http://www . ceremade.dauphine.fr/ blanchet/ (Adrien Blanchet), http://www. ceremade.dauphine.fr/ dolbeaul/ (Jean Dolbeault), http://www . capde.cl/members/robusg (Michał Kowalczyk). 
of the domain in higher dimensional models, see for instance [14], or applications of molecular motors in biology, see for instance [15-17], will not be addressed here.

In $[18,19]$, the effective diffusion constant is computed by a method which differs from ours, based on statistical fluctuations. Also see [20] for some earlier considerations, and [21] for the computation of a generalized Einstein relation. The relation between the diffusion coefficient and the mobility of a Brownian particle in a tilted periodic potential is studied in [22]. The ratio $\mathrm{k}_{\mathrm{B}} \Theta$ of the diffusion constant $\kappa_{\omega}$ to the differential mobility $\mu_{\omega}$ is in general not equal to the temperature of the environment (multiplied by Boltzmann's constant $k_{B}$ ). The physical meaning of $\Theta$ far from equilibrium is analyzed in [23] and interpreted as an effective temperature in the large scale description of the system so that the relation $\kappa_{\omega}=\mu_{\omega} \mathrm{k}_{\mathrm{B}} \Theta$ can be interpreted as an extension of Einstein's relation. An interesting experiment for measuring the violation of Einstein's relation can be found in [24]. See Section 5.1 for more details.

Experimental measurements of the drift velocity corresponding to the diffusion of colloidal particles in presence of optical traps and a detailed explanation of the method can be found in [25], with abundant theoretical justifications. Some of the qualitative features were at least partially known before, see, e.g., [26]. In [27-29], the analysis of [25] is refined and emphasis is put not only on the computation of the drift velocity, but also on the effective diffusion constant. Interestingly, the authors of [30] favorably evaluate the possibility of using tilted ratchet mechanisms to implement separation of two types of filaments of DNA.

The simplest version of the stochastic Stokes' drift model describes a density $f(t, x)$ of particles obeying to the equation

$$
f_{t}=f_{x x}+\left(\psi^{\prime}(x-\omega t) f\right)_{x}
$$

where $f_{t}$ and $f_{x}$ denote derivatives with respect to the time $t \geq 0$ and the position $x$ respectively, and $\psi^{\prime}(x-\omega t)$ is a traveling potential moving at constant speed $\omega$. We assume that the function $\psi$ is 1 -periodic: $\psi(x+1)=\psi(x)$, and consider the unbounded problem, $x \in \mathbb{R}$. At $t=0, f(0, \cdot)=f_{0}$ is a given smooth probability distribution, so that, by conservation of mass, $\int_{\mathbb{R}} f(t, x) d x=1$ for any $t \geq 0$. The question we investigate in this paper is the behavior of $f$ for large values of $t$. Our main results can be summarized as follows.

(1) Denote by $\bar{x}(t):=\int_{\mathbb{R}} x f(t, x) d x$ the position of the center of mass. There exists a drift velocity $c_{\omega}$ which is characterized in Section 2 such that, for some $\gamma>0$, $\left|\frac{d}{d t} \bar{x}(t)-c_{\omega}\right|=O\left(e^{-t / \gamma}\right)$ as $t \rightarrow \infty$.

(2) A diffusive traveling front appears. In the reference frame attached to the center of mass, the solution converges, in self-similar variables, to a Gaussian, modified by a highly oscillating perturbation. If we introduce the scale $R(t):=\sqrt{1+2 t}$ and consider 
the change of variables

$$
f(t, x)=\frac{1}{R(t)} u\left(\log R(t), \frac{x-c_{\omega} t}{R(t)}\right)
$$

then, in the new variables, with $R=e^{t}$ and $z=R x-\frac{1}{2}\left(R^{2}-1\right)\left(\omega-c_{\omega}\right), u$ is a solution of

$$
u_{t}=u_{x x}+(x u)_{x}+R\left(\left(\psi^{\prime}(z)+c_{\omega}\right) u\right)_{x}
$$

and we shall prove by an asymptotic expansion, see Section 3, that

$$
u(t, x)=g_{\omega}(z) h-\frac{1}{R} g_{\omega}^{(1)}(z) h_{x}+O\left(R^{-2}\right) .
$$

Here $g_{\omega}$ is a periodic solution of a tilted ratchet problem, see Section 2, which depends on the fast oscillating variable $z$. The profile $h=h(t, x)$ is the solution of an effective Fokker-Planck equation

$$
h_{t}=\kappa_{\omega} h_{x x}+(x h)_{x},
$$

where the diffusion coefficient $\kappa_{\omega}$ is determined in terms of $g_{\omega}^{(1)}$, which is again given by an ordinary differential equation. Let $h_{\infty}$ be the unique stationary solution of (4) such that $\int_{\mathbb{R}} h_{\infty} d x=1$ and define $u_{\infty}(t, x):=g_{\omega}(z) h_{\infty}(x)$. For any $\delta>0$ arbitrarily small, there is a positive constant $C_{\delta}$, depending on $\psi$ and $f_{0}$, such that

$$
\int_{\mathbb{R}}\left|u(t, x)-u_{\infty}(t, x)\right| d x \leq C_{\delta} e^{-t / \tau}
$$

for any $t \geq 0$, where $\tau \geq 2 \kappa_{\omega} / \kappa_{0}+\delta$. Moreover, we have good reasons to conjecture that the optimal possible value of $\tau$ is $2 \kappa_{\omega} / \kappa_{0}$, and $\kappa_{\omega} / \kappa_{0}>1$ for any $\omega>0$. At least we can prove this last property in the limiting regimes $\omega \rightarrow 0$ and $\omega \rightarrow \infty$.

We observe that in the original time scale the rate of convergence in (5) is like $t^{-1 / \tau}$, where in general we expect that $\tau>2$. This means that in the dynamics of the stochastic Stokes' drift two time scales have to be distinguished. First, periodic modulations over an initial profile appear without any appreciable drift - this process is very fast since the modulations settle exponentially fast. Second, developed, modulated profiles evolve on a much slower, algebraic time scale, approaching (from a macroscopic point of view) a gaussian traveling front.

(3) We establish various properties of $c_{\omega}$ and $\kappa_{\omega}$ in Sections 3 and 5 . For instance, we prove that the front travels asymptotically at speed $c_{\omega}$ and give a formula for $c_{\omega} / \omega$, for small values of $\omega$. We also obtain that $c_{\omega}$ converges to 0 as $\omega \rightarrow \infty$, hence showing that 
there is always a maximal value of $c_{\omega}$ for some finite value of $\omega$. Even more interesting is the characterization of the dynamics of the front, which has been observed only in Monte-Carlo simulations. Computations are usually noisy while we provide exact identities, which turn out to be easy to study qualitatively and numerically. The diffusion at large scale is governed by the effective diffusion coefficient $\kappa_{\omega}$. For small values of $\omega, \kappa_{\omega}$ is always less than 1 , thus showing a narrowing of the front, but in our examples we numerically observe that $\kappa_{\omega}>\kappa_{0}$, hence inducing a slower rate of convergence in self-similar variables. We strongly suspect that the smallest value of $\kappa_{\omega}$ is always achieved for $\omega=0$. In the large $\omega$ regime, $\kappa_{\omega}$ is anyway larger than 1 , which results in a front expanding faster (or fatter in self-similar variables), but also gives a lower rate of convergence of the relative entropy corresponding to $2 / \tau \leq \kappa_{0} / \kappa_{\omega}<1$.

(4) The efficiency of the transport has already been studied numerically in various contributions. We define a new dimensionless number, the efficiency,

$$
\mathrm{E}:=\frac{c_{\omega}^{2}}{\omega \kappa_{\omega}}
$$

which is well adapted to our model and measures accurately the coherent transport at least in the two examples of this paper. Details are given in Section 4.

\section{From stochastic Stokes' drift to a tilted ratchet model}

We first observe that the stochastic Stokes' drift is analogous to a simple Brownian ratchet mechanism. Actually, if $f$ is a solution of $(1)$, we observe that $\tilde{f}(t, x)=$ $f(t, x-\omega t)$ is a solution of

$$
\tilde{f}_{t}=\tilde{f}_{x x}+\left(\left(\omega+\psi^{\prime}\right) \tilde{f}\right)_{x}
$$

a problem which is known as the tilted Smoluchowski-Feynman ratchet, see for instance [12]. Tilted Brownian ratchets are actually much more general, since in the equation for $\tilde{f}, \psi$ may still depend on $t$. An important effect in such models is the notion of flow reversals, see for instance [31-34,13,35]. In our case we conjecture that such a flow reversal is impossible, a property that we shall observe on examples and which is reflected by the fact that $\omega$ and $c_{\omega}$ have the same signs.

On large time scales, the constant drift term, $\omega \tilde{f}_{x}$, is responsible for a displacement of the center of mass, but the solution is also spreading on a large number of periods of $\psi$. It is therefore natural to expect that the speed of the center of mass is determined by the flux of mass through one cell of period $\ell=1$, supplemented with periodic conditions. This can be made rigorous by the following folding transformation as 
follows. Consider the doubly periodic problem

$$
\left\{\begin{array}{l}
g_{t}=g_{x x}+\left(\left(\omega+\psi^{\prime}\right) g\right)_{x} \\
g(t=0, x)=g_{0}(x)=\sum_{k \in \mathbb{Z}} f_{0}(x+k),
\end{array}\right.
$$

for which we assume that $g(t, x+1)=g(t, x)$ for any $x \in \mathbb{R}$ and any $t \geq 0$. By linearity of the equations (see [12] for more details), we get

$$
g(t, x)=\sum_{k \in \mathbb{Z}} f(t, x-\omega t+k) \quad \forall(t, x) \in \mathbb{R}^{+} \times(0,1) .
$$

Using either the Poincaré or the logarithmic Sobolev inequality, it is easy to prove that $g$ converges exponentially fast (in $L^{2}$ and $L^{1}$ norms, respectively) to a stationary periodic solution $g_{\omega}$ of (6), which is unique by a contraction property, and solves

$$
\left(g_{\omega}\right)_{x x}+\left(\left(\omega+\psi^{\prime}\right) g_{\omega}\right)_{x}=0
$$

with periodic boundary conditions. If we take a primitive of $(7)$, we get that $x \mapsto$ $\left(g_{\omega}\right)_{x}+\left(\omega+\psi^{\prime}\right) g_{\omega}=: A(\omega)$ is constant. By taking one more integral, using the normalization condition $\int_{0}^{1} g_{\omega}(x) d x=1$ and the definition of $c_{\omega}$, we get that

$$
\omega-c_{\omega}=\omega \int_{0}^{1} g_{\omega} d x+\int_{0}^{1} \psi^{\prime} g_{\omega} d x=A(\omega) .
$$

Equation (7) can be semi-explicitly solved as

$$
g_{\omega}(x)=e^{-\omega x-\psi(x)}\left(B(\omega)+A(\omega) \int_{0}^{x} e^{\omega y+\psi(y)} d y\right)
$$

where $B(\omega)$ is determined by the conditions $\int_{0}^{1} g_{\omega} d x=1$ and $g_{\omega}(0)=g_{\omega}(1)$. More precisely, with

$$
\begin{array}{ll}
\alpha(\omega):=e^{\omega}-1 & \beta(\omega):=\int_{0}^{1} e^{\omega x+\psi(x)} d x \\
\gamma(\omega):=\int_{0}^{1} e^{-\omega x-\psi(x)} d x & \delta(\omega):=\int_{0}^{1} \int_{0}^{x} e^{\omega y+\psi(y)-\omega x-\psi(x)} d y d x
\end{array}
$$

we obtain

$$
A(\omega)=\frac{\alpha(\omega)}{\alpha(\omega) \delta(\omega)+\beta(\omega) \gamma(\omega)} \quad \text { and } \quad B(\omega)=\frac{\beta(\omega)}{\alpha(\omega) \delta(\omega)+\beta(\omega) \gamma(\omega)} .
$$


These results are classical, see for instance [36-38] for more details. As a consequence, we obtain a semi-explicit formula for $c_{\omega}$, namely

$$
c_{\omega}=\omega-A(\omega)
$$

We will now illustrate our results in the case of $\psi(x)=\sin (2 \pi x)$ (sinusoidal case) and of an asymmetric smooth sawtooth potential, see Fig. 1. Notice that in the tilted ratchet point of view, the current is $A(\omega)$. It is actually very interesting to compare $c_{\omega}$
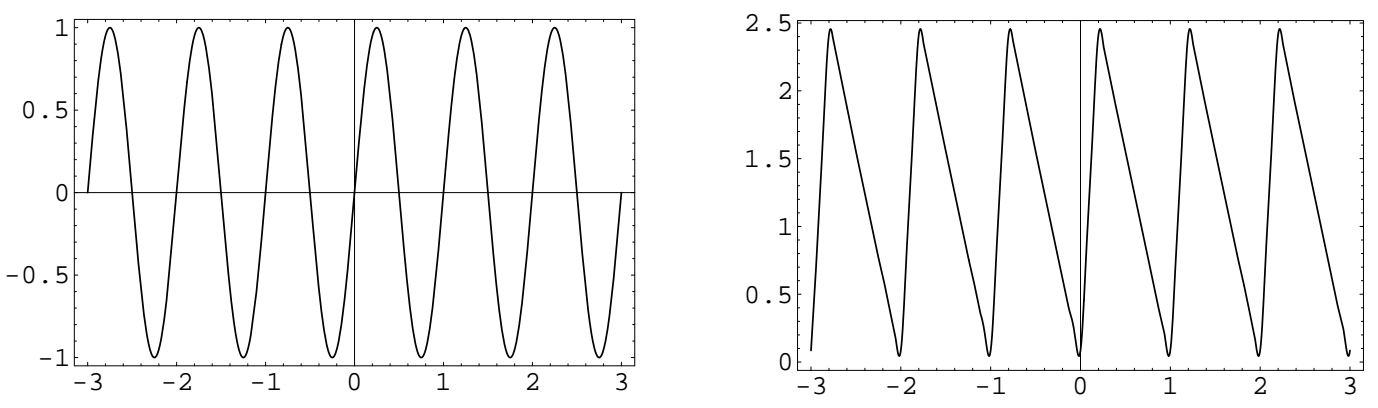

Fig. 1. Plots of the potential $\psi$ in the sinusoidal case (left) and in the asymmetric smooth sawtooth potential (right), which is computed here as a truncated Fourier series of $x \mapsto x / x_{0}$ if $x<x_{0}$ and $x \mapsto(1-x) /\left(1-x_{0}\right)$ if $x>x_{0}$, with $x_{0}=0.2$.

with the asymptotic drift velocity $c_{\omega}^{0}$ when there is no diffusion. See [38] for similar considerations. The solutions of $f_{t}=\left(\psi^{\prime}(x-\omega t) f\right)_{x}$ are easily solved by considering the equations of the characteristics, $\frac{d x}{d t}=-\psi^{\prime}(x(t)-\omega t)$. Let $y(t):=x(t)-\omega t$ and consider the corresponding equation $\frac{d y}{d t}=-\psi^{\prime}(y)-\omega$. For $\omega>0$, there are two main regimes:

(i) Case $0<\omega<\max _{[0,1]} \psi^{\prime}$ : any solution $t \mapsto y(t)$ converges to a local minimum of the function $y \mapsto \omega y-\psi(y)$, and so

$$
c_{\omega}^{0}:=\lim _{t \rightarrow \infty} \frac{x(t)}{t}=\omega
$$

(ii) Case $\omega>\max _{[0,1]} \psi^{\prime}$ : we observe that $\tau(\omega):=\int_{y(t)}^{y(t)+1} \frac{d x}{\omega+\psi^{\prime}(x)}$ does not depend on $t$, and so $y(t) \sim-t / \tau(\omega)$ as $t \rightarrow \infty$. It follows that

$$
\tau(\omega)=\int_{0}^{1} \frac{d x}{\omega+\psi^{\prime}(x)} \quad \text { and } \quad c_{\omega}^{0}:=\lim _{t \rightarrow \infty} \frac{x(t)}{t}=\omega-\frac{1}{\tau(\omega)} .
$$

A characteristic property of the curve $\omega \mapsto c_{\omega}^{0}$ is the critical tilt: the discontinuity of the derivative separates the two regimes. The curve $\omega \mapsto c_{\omega}$ is a smoothed version of $\omega \mapsto c_{\omega}^{0}$. When $\psi$ is not symmetric, asymmetry effects are present when $\omega$ is replaced by $-\omega$, as shown in the case of the asymmetric smooth sawtooth potential. See Fig. 2. 

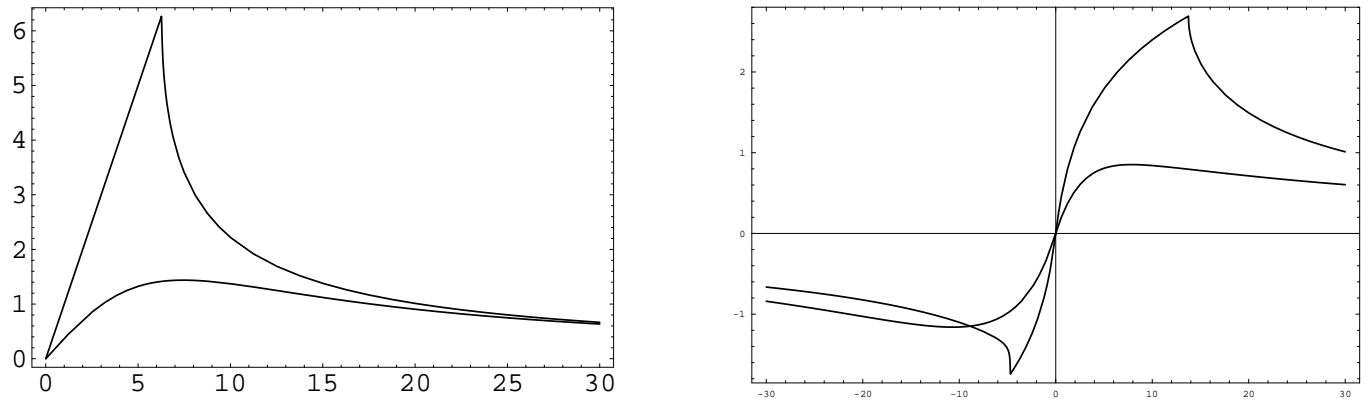

Fig. 2. Plots of $c_{\omega}$ and $c_{\omega}^{0}$ as functions of $\omega$ in the sinusoidal case (left) and in the case of the asymmetric smooth sawtooth potential (right, in logarithmic coordinates: $\omega \mapsto \log \left(1+c_{\omega}\right)$ for $\omega>0)$. In the sinusoidal case, the symmetry is reflected by the fact that $c_{-\omega}=-c_{\omega}$ (values corresponding to $\omega<0$ are not represented). This is not true in the sawtooth case.

\section{The diffusive traveling front}

Consider now a solution $f$ of $(1)$, with the normalization condition $\int_{\mathbb{R}} f_{0} d x=1$ and let $\bar{x}(t):=\int_{\mathbb{R}} x f(t, x) d x$ be the position of the center of mass. An integration by parts and a change of variables show that

$$
\begin{aligned}
\frac{d \bar{x}}{d t}=\int_{\mathbb{R}} x f_{t} d x & =-\int_{\mathbb{R}} \psi^{\prime}(x-\omega t) f(t, x) d x=-\sum_{k \in \mathbb{Z}} \int_{0}^{1} \psi^{\prime}(x-\omega t) f(t, x+k) d x \\
& =-\int_{0}^{1} \psi^{\prime}(x) g(t, x) d x \underset{t \rightarrow \infty}{\sim}-\int_{0}^{1} \psi^{\prime}(x) g_{\omega}(t, x) d x=: c_{\omega}
\end{aligned}
$$

and a more careful analysis of (6) even proves that $\frac{d \bar{x}}{d t}-c_{\omega}$ converges at an exponential rate. Hence

$$
\bar{x}(t) \sim c_{\omega} t \quad \text { as } \quad t \rightarrow \infty
$$

Because of (8), it makes sense to introduce the change of coordinates (2), in order to understand the large time behaviour of $f$. In the new variables, the equation for $u$ is (3). Let us introduce a two-scale function $\mathrm{U}$ such that

$$
u(t, x)=\mathrm{U}(t, x ; z)
$$

with $R=e^{t}$ and $z=R x-\frac{1}{2}\left(R^{2}-1\right)\left(\omega-c_{\omega}\right)$, in order to investigate the large limit. Using the chain rule, we can write an equation for $U$ which is equivalent to (3), namely

$$
R^{2} \mathrm{~L}_{0} \mathrm{U}+R \mathrm{~L}_{1} \mathrm{U}+\mathrm{L}_{2} \mathrm{U}=0
$$

with 


$$
\begin{aligned}
& \mathrm{L}_{0} \mathrm{U}:=\mathrm{U}_{z z}+\left(\left(\omega+\psi^{\prime}(z)\right) \mathrm{U}\right)_{z} \\
& \mathrm{~L}_{1} \mathrm{U}:=\left(2 \mathrm{U}_{z}+\left(\psi^{\prime}(z)+c_{\omega}\right) \mathrm{U}\right)_{x} \\
& \mathrm{~L}_{2} \mathrm{U}:=\mathrm{U}_{x x}+(x \mathrm{U})_{x}-\mathrm{U}_{t}
\end{aligned}
$$

and make a formal asymptotic expansion in which we solve the equation order by order in powers of $R$ for a solution, for which we make the ansatz $\mathrm{U}=\mathrm{U}_{0}+R^{-1} \mathrm{U}_{1}+R^{-2} \mathrm{U}_{2}$. At order $R^{2}$, we find $\mathrm{L}_{0} \mathrm{U}_{0}=0$ that is solved by $\mathrm{U}_{0}(t, x ; z)=g_{\omega}(z) h(t, x)$, where $g_{\omega}$ is the stationary solution of the tilted ratchet equation (7). At order $R$ we get

$$
\mathrm{L}_{0} \mathrm{U}_{1}+\mathrm{L}_{1} \mathrm{U}_{0}=0
$$

This gives $\mathrm{U}^{(1)}(t, x ; z)=g_{\omega}^{(1)}(z) h_{x}(t, x)$, where $g_{\omega}^{(1)}$ is a solution of

$$
\left(g_{\omega}^{(1)}\right)_{z z}+\left(\left(\omega+\psi^{\prime}\right) g_{\omega}^{(1)}\right)_{z}=-2\left(g_{\omega}\right)_{z}-\left(\psi^{\prime}+c_{\omega}\right) g_{\omega} .
$$

Recall that we look for solutions that are periodic in the $z$ variable. A necessary and sufficient condition for the existence of a solution to the above equation is the fact that the average on $(0,1)$ of the right hand side of $(10)$ is 0 . Since all functions are periodic and $\int_{0}^{1} g_{\omega}(z) d z=1$, we recover the definition of $c_{\omega}$. Notice that $g_{\omega}^{(1)}$ is unique up to the addition of a multiple of $g_{\omega}$, so we may further assume that $\int_{0}^{1} g_{\omega}^{(1)} d z=0$. If we stop the expansion at order $R^{0}=1$, we obtain

$$
\mathrm{L}_{0} \mathrm{U}_{2}+\mathrm{L}_{1} \mathrm{U}_{1}+\mathrm{L}_{2} \mathrm{U}_{0}+\frac{1}{R}\left(\mathrm{~L}_{1} \mathrm{U}_{2}+\mathrm{L}_{2} \mathrm{U}_{1}\right)+\frac{1}{R^{2}} \mathrm{~L}_{2} \mathrm{U}_{2}=0 .
$$

However, if we look at the terms of order $R^{0}=1$, a solvability condition results from the integration of (9) with respect to $z$. We obtain that $\int_{0}^{1}\left(\mathrm{~L}_{1} \mathrm{U}_{1}+\mathrm{L}_{2} \mathrm{U}_{0}\right) d z=0$, which shows that the function $h$ solves the modified Fokker-Planck equation (4) for the effective profile $h$, where the effective diffusion coefficient is given by

$$
\kappa_{\omega}:=1+\int_{0}^{1} \psi^{\prime}(z) g_{\omega}^{(1)}(z) d z
$$

Any solution of (4) with $\int_{\mathbb{R}} h(0, x) d x=1$ converges to a Gaussian, $h_{\infty}$, exponentially in $L^{2}$ and $L^{1}$ norms. Therefore, at first order, $u(t, x)$ behaves for large values of $t$ like

$$
u_{\infty}(t, x)=g_{\omega}(z) h_{\infty}(x), \quad h_{\infty}(x):=\frac{e^{-\frac{|x|^{2}}{2 \kappa_{\omega}}}}{\sqrt{2 \pi \kappa_{\omega}}}
$$

where $z=e^{t} x-\frac{1}{2}\left(e^{2 t}-1\right)\left(\omega-c_{\omega}\right)$. Using relative entropies and homogenized logarithmic Sobolev inequalities, one can then prove (5). The function $u_{\infty}$ therefore 
describes the asymptotic regime of $u$, in self-similar, traveling variables. In the original variables, $f_{\infty}(t, x)=\frac{1}{R} u_{\infty}\left(\log R, \frac{x-c_{\omega} t}{R}\right)$ with $R(t):=\sqrt{1+2 t}$ describes the intermediate asymptotics of the solution of (1). It is highly oscillatory, with an effective profile given by $F_{\infty}(t, x):=\frac{1}{R(t)} h_{\infty}\left(\frac{x-c_{\omega} t}{R(t)}\right)$, which is the diffuse, traveling front. More details on this asymptotic expansion and rigorous proofs can be found in [2]. See Fig. 3.
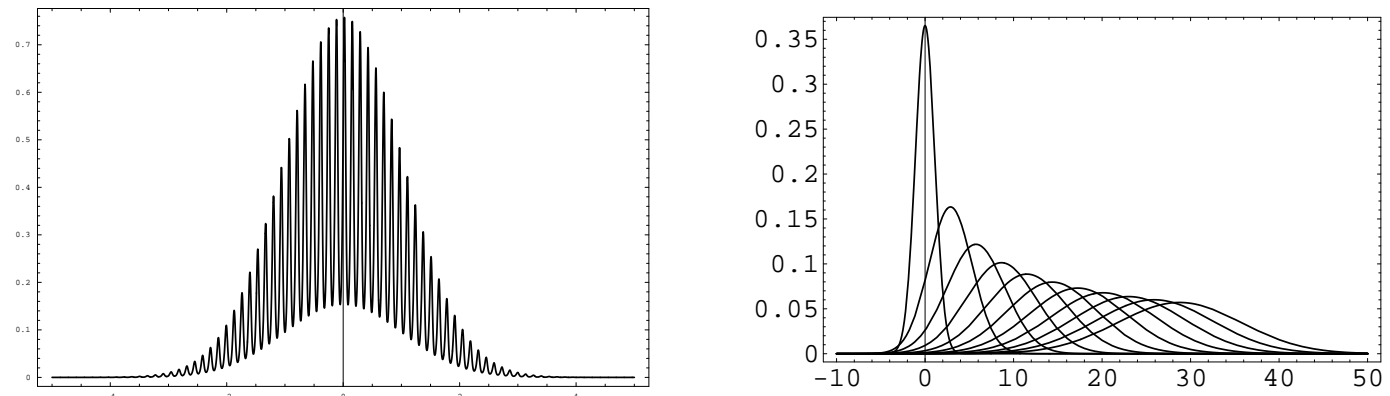

Fig. 3. In the sinusoidal case, the limiting function $u_{\infty}$ is shown on the left, in self-similar-variables, while on the right, the diffuse, traveling front $F_{\infty}$ is plotted in the original variables for $t=0,1, \ldots 20$. Here we take $\omega=5$ and (left) $u_{\infty}(t, x)$ is shown as a function of $x$ for $t=2$.

The effective diffusion coefficient $\kappa_{\omega}$ is a global, macroscopic quantity, which should not be confused with the local effective diffusion constant which appears in some papers, see $[37,39]$. It plays two roles:

(1) The effective diffusion coefficient $\kappa_{\omega}$ determines the variance of the Gaussian function $h_{\infty}$ and therefore controls the size of the traveling font. A pure diffusion $f_{t}=f_{x x}$ would give rise to a self-similar Gaussian function $(4 \pi t)^{-1 / 2} e^{-|x|^{2} /(4 t)}$, and so $\kappa_{\omega}$ has to be compared with 1 . When $\kappa_{\omega}<1$, the front is more peaked than what we would get from a pure diffusion, while on the opposite, $\kappa_{\omega}>1$ corresponds to a front which is diffusing faster. See Section 5 for some partial, rigorous results.

(2) In (5), the functional inequality:

$$
\int_{\mathbb{R}} v \log \left(\frac{v}{u_{\infty}}\right) d x \leq C(t) \int_{\mathbb{R}}\left|\frac{v_{x}}{v}-\frac{\left(u_{\infty}\right)_{x}}{u_{\infty}}\right|^{2} v d x,
$$

holds for any function $v$, for some $C(t)>0$ such that $\lim _{t \rightarrow \infty} C(t)=\tau / 2$. It is known, see [2], that $\tau \geq 2 \kappa_{\omega} / \kappa_{0}$. The above homogenized logarithmic Sobolev inequality is a limit case of a family of generalized Poincaré inequalities for which the optimal constant converges as $t \rightarrow \infty$ to $2 \kappa_{\omega} / \kappa_{0}$. It is therefore natural to conjecture that the optimal possible value of $\tau$ is also $\tau=2 \kappa_{\omega} / \kappa_{0}$, at least for a large class of potentials $\psi$, but the question is still mathematically open. Now, if $\kappa_{\omega} / \kappa_{0} \geq 1$, then $\tau=2 \kappa_{\omega} / \kappa_{0}$ governs the rate of convergence in (5). If $\kappa_{\omega} / \kappa_{0}<1$, other terms of order $e^{-t}$, which means $O(1 / \sqrt{t})$ in the original variables, would eventually dominate the convergence process. This last case is never observed numerically. 

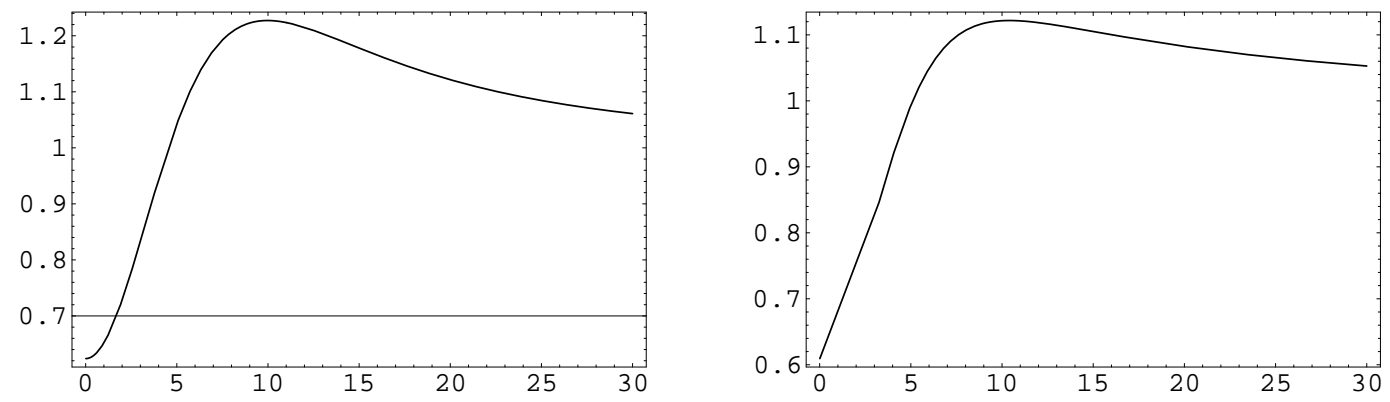

Fig. 4. Plot of the diffusion coefficient $\kappa_{\omega}$ as a function of $\omega$ in the sinusoidal case (left) and in the smooth sawtooth potential case (right).

\section{Measuring the efficiency of coherent transport}

Measuring the efficiency of Brownian motors is a tricky issue. It requires specific tools. We may refer for instance to [35] for a recent reference in this direction, with some numerical simulations. Also see [7, Fig. 2] for an early result in the context of the stochastic Stokes' drift, [40] for recent simulations corresponding to a simple model, and [41] for detailed considerations on transport coherence and values of the Péclet number. In the very simple model considered in this paper, there are only few available parameters. As explained in $[41,40]$, the Péclet number Pe describes the competition between the directional drift and the stochastic diffusion of the particle. It is defined, with our notations, by

$$
\mathrm{Pe}:=\frac{c_{\omega} \ell}{\kappa_{\omega}}
$$

where $\ell$ is a typical length scale. One can easily check that this is a dimensionless number. Larger Pe number means that the drift predominates over diffusion and there is high transport coherence. In other words, the effective distribution is transported far away from the original data and stays peaked around its maximum value, at least when the variance is measured in the same units as the displacement of the center of mass.
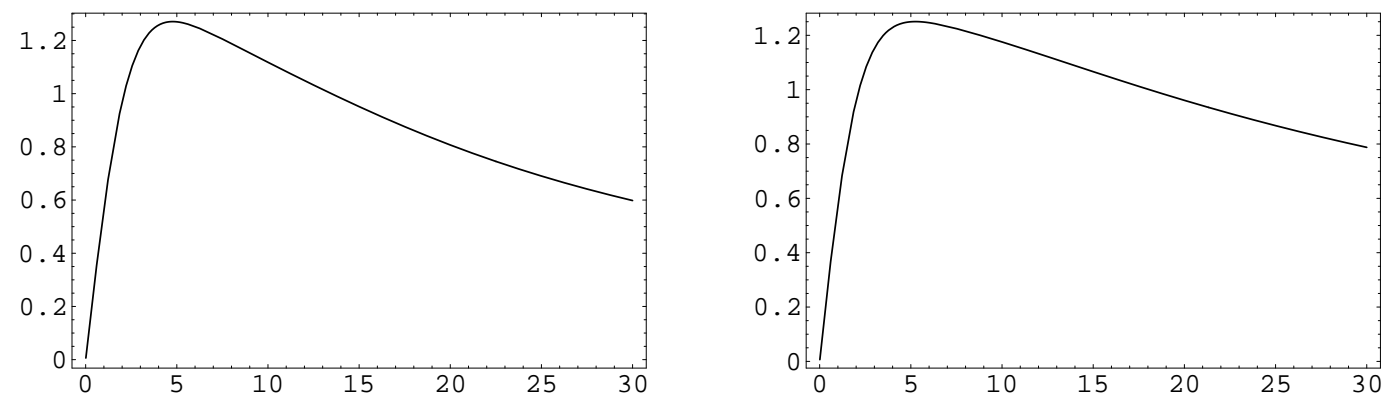

Fig. 5. Plot of the Péclet number Pe as a function of $\omega$ in the sinusoidal case (left) and in the smooth sawtooth potential case (right). 
In $[41,40]$, the typical length scale is the period of the potential $\psi$, that is $\ell=1$ in our notations, and it is suggested that a criterion for efficient transport, preserving simultaneously the coherence of the distribution and optimizing its displacement, is $\mathrm{Pe}>2$. This criterion does not make much sense for a study of the large time behavior, as the variance of the distribution, which is of diffusive nature and grows like $\sqrt{\kappa_{\omega} t}$, is always dominated by the displacement, which is linear and of the order of $c_{\omega} t$, when $t$ is large. To remedy this, we can suggest the following analysis. We may first use the Péclet number to define a characteristic length scale

$$
\mathrm{L}:=\frac{\ell}{\mathrm{Pe}}
$$

Recall that with our notations, $\ell=1$. The value of $\mathrm{L}$ corresponds to the displacement of the center of mass for which this displacement is equal to the variance of the effective distribution. If the effective distribution is originally centered at zero and evolves according to (4) up to a translation at constant velocity $c_{\omega}$, this occurs for $t=\mathrm{T}$ such that $\sqrt{\kappa_{\omega} \mathrm{T}}=c_{\omega} \mathrm{T}=\mathrm{L}$, and at that time, the percentage of the initial distribution which is still in the $x<0$ region is given by $\int_{-\infty}^{0} \exp \left[-|x-\mathrm{L}|^{2} /\left(2 \kappa_{\omega} \mathrm{T}\right)\right] d x=\frac{1}{2} \operatorname{Erf}(1 / \sqrt{2}) \approx 16 \%$. See Fig. 6 .

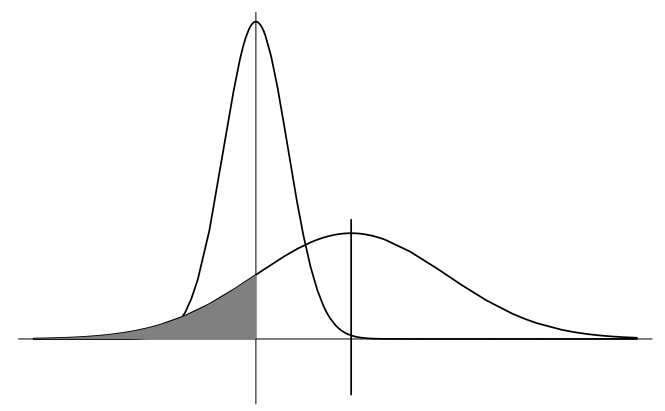

Fig. 6. Definition of $\mathrm{L}$ and $\mathrm{T}$ can be understood as follow. If one starts with a Gaussian distribution centered at $x=0$ and evolve it according to (4), $\mathrm{T}$ is the time for which the solution (centered at $\mathrm{L}$ in the above plot) has a variance equal to $\mathrm{L}$. The grey area represents $16 \%$ of the area below the solution at time $t=\mathrm{T}$.

Now we may observe that in the above discussion, we have also introduced a characteristic time scale $\mathrm{T}=\kappa_{\omega} / c_{\omega}^{2}$ which is related with the Péclet number by the formula

$$
\mathrm{T}=\frac{\ell}{c_{\omega} \mathrm{Pe}}
$$

It turns out that the stochastic Stokes' drift has a natural time scale, which is the time period of the potential $T_{0}:=\ell / \omega$. Hence it is meaningful to consider

$$
\mathrm{N}:=\frac{\mathrm{T}}{\mathrm{T}_{0}}=\frac{\omega \kappa_{\omega}}{\ell c_{\omega}^{2}}=\frac{\omega}{c_{\omega} \mathrm{Pe}}
$$


which measures the time in takes to achieve the equality $\sqrt{\kappa_{\omega} \mathrm{T}}=c_{\omega} \mathrm{T}$ in natural units, and to define the efficiency of the transport by

$$
\mathrm{E}:=\frac{1}{\mathrm{~N}}=\frac{\ell c_{\omega}^{2}}{\omega \kappa_{\omega}}=\operatorname{Pe} \frac{c_{\omega}}{\omega}
$$
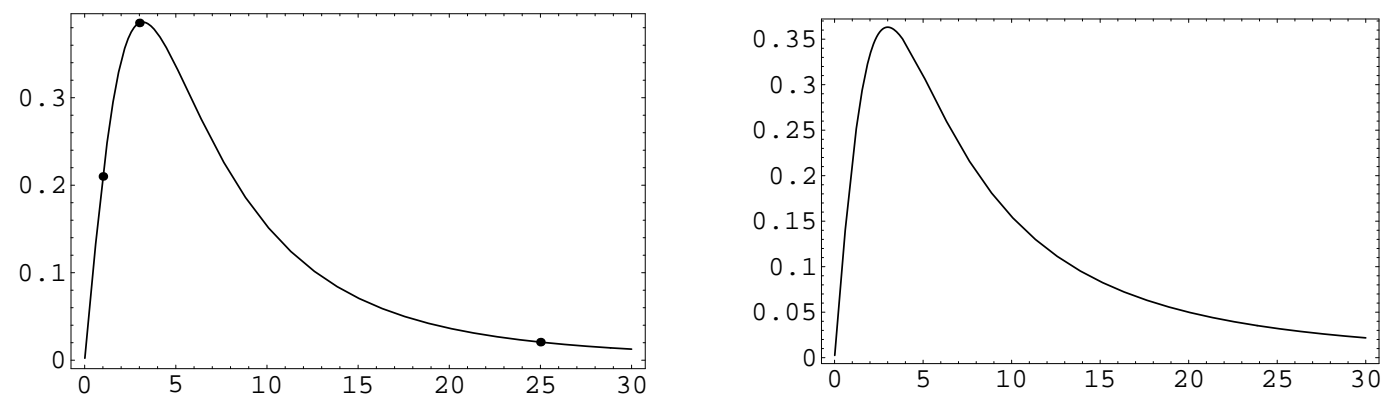

Fig. 7. Plot of the efficiency $\mathrm{E}$ as a function of $\omega$ in the sinusoidal case (left) and in the smooth sawtooth potential case (right). We observe that in both cases, the maximum is extremely well defined. Dots (left) correspond $(\omega, \mathrm{E}(\omega))$ taking the values $(1,0.210),(3,0.385)$, $(25,0.021)$ and will be reused in Fig. 8.
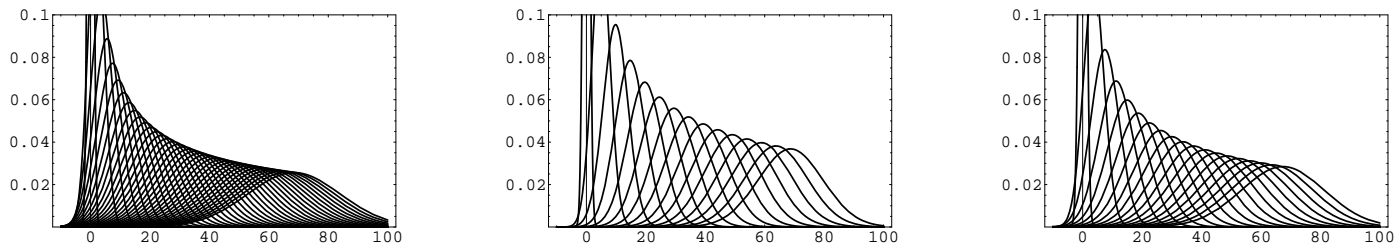

Fig. 8. The effective profile $F_{\infty}$ is represented for $\omega$ taking the values 1,3 and 25 , which correspond to the dots in Fig. 7 (left). Curves are plotted for $\omega=1$ (left), 3 (center), 25 (right) for $t=0,5,10$, etc, as long as $c_{\omega} t \leq 70$. The curve corresponding to $\omega=3$ (center) is the most efficient, in the sense that $c_{\omega} t \approx 70$ is reached for a smaller value of $t$ than for the other curves and the solution is kept more peaked. Computations are done in the case of the sinusoidal potential.

The strength of our approach is that by our asymptotic expansion, we have been able to identify $\kappa_{\omega}$ and we have a formula which allows us to plot it precisely, see Fig. 5. The shapes of the curves $\omega \mapsto c_{\omega}$ and $\omega \mapsto \kappa_{\omega}$ combine well to define an optimum of the efficiency, characterized by the dimensionless number $\mathrm{E}$, which reflects the idea of coherent transport.

\section{Drift velocity and homogenized diffusion coefficient: further results}

In this last section, we list some qualitative properties that can be established analytically and are of general interest. We also formulate a few conjectures which should be true for any type of potential $\psi$. 


\subsection{Mobility and Einstein's relation}

In the tilted ratchet picture, $\omega$ is proportional to the applied force $\mathrm{F}$ :

$$
\omega=\frac{1}{\eta} \mathrm{F}
$$

where $\eta$ is the viscous friction coefficient, has the dimension of the inverse of a time, and takes value 1 in our units. The mobility is defined by

$$
\mu_{\omega}:=\frac{c_{\omega}}{\mathrm{F}}=\frac{c_{\omega}}{\eta \omega}
$$

so we can write

$$
\mathrm{E}=\mathrm{Pe} \frac{c_{\omega}}{\omega}=\eta \mu_{\omega} \mathrm{Pe}
$$

It has been argued that Einstein's relation

$$
\frac{\kappa_{\omega}}{\mu_{\omega}}=\mathrm{k}_{\mathrm{B}} \Theta
$$

defines a notion of effective temperature $\Theta$. Here $k_{B}$ is Boltzmann's constant. As can be seen on Fig. $9, \Theta$ is not constant in terms of $\omega$.
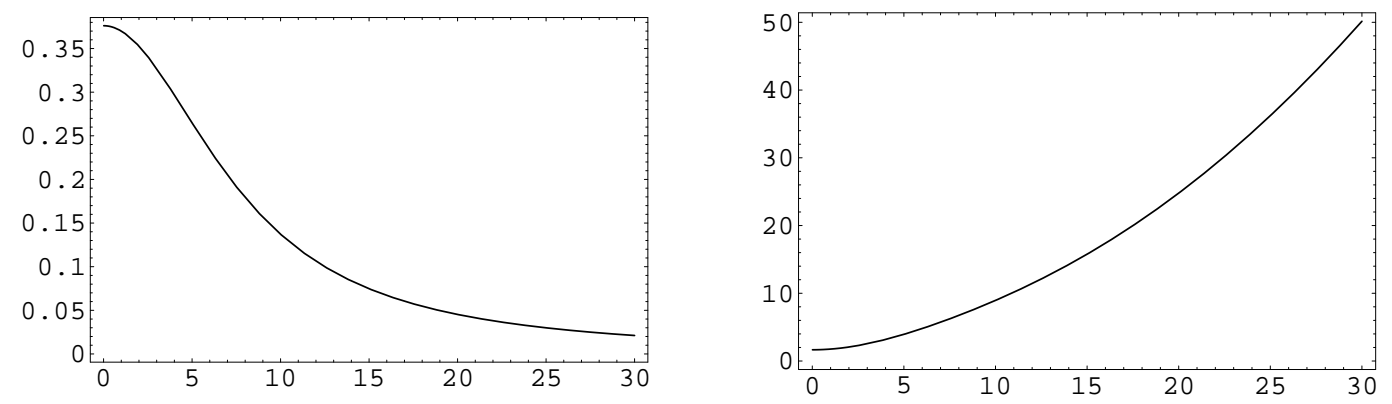

Fig. 9. Plot of the mobility $\mu_{\omega}$ (left) and the effective temperature $\Theta$ (measured in units of $1 / \mathrm{k}_{\mathrm{B}}$ ) of the tilted Brownian ratchet for values of $\omega$ ranging between 0 and 30 in the sinusoidal case.

\subsection{The stationary solution of the tilted ratchet problem}

The 1 -periodic function $g_{\omega}$ which solves (7) and is normalized by the condition $\int_{0}^{1} g_{\omega} d z=1$, has the following properties:

(1) $g_{\omega}$ is bounded from below by a positive constant, uniformly with respect to $\omega$. 
(2) In the limit case $\omega=0$, we have: $g_{0}=e^{-\psi} / \int_{0}^{1} e^{-\psi} d z$.

(3) As $\omega \rightarrow \infty, g_{\omega}$ uniformly converges to 1 .
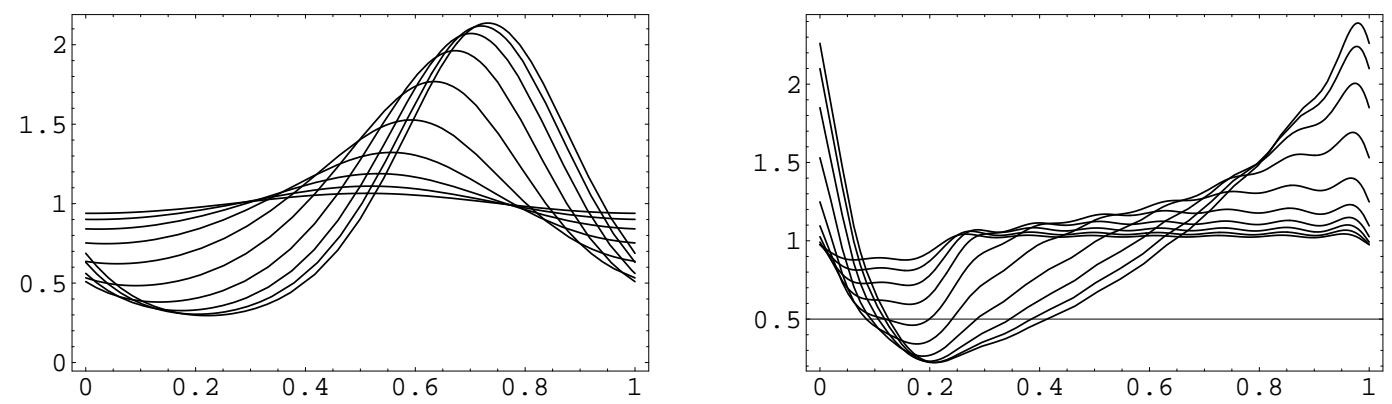

Fig. 10. Plot of the stationary solution $g_{\omega}$ of the tilted Brownian ratchet for increasing values of $\omega$ ranging between 1 and 100 (logarithmic scale) in the sinusoidal case (left) and in the smooth sawtooth potential case (right).

\subsection{Drift velocity}

When there is no diffusion, for large values of $\omega$, an asymptotic expansion of $c_{\omega}^{0}$ as defined in Section 2 gives

$$
c_{\omega}^{0}=\frac{1}{\omega} \int_{0}^{1}\left|\psi^{\prime}\right|^{2} d x+O\left(\frac{1}{\omega^{2}}\right) .
$$

In presence of diffusion, for any $\omega>0$, we have $c_{\omega}<\omega$,

$$
\lim _{\omega \searrow 0} \frac{c_{\omega}}{\omega}=1-\frac{1}{\int_{0}^{1} e^{\psi} d z \int_{0}^{1} e^{-\psi} d z} \quad \text { and } \quad \lim _{\omega \nearrow \infty} c_{\omega}=0 .
$$

We numerically observe that $c_{\omega}$ is always positive for positive values of $\omega$.

\subsection{Effective diffusion coefficient}

(1) We numerically observe that the smallest value of the effective diffusion coefficient is achieved at $\omega=0: \kappa_{\omega}>\lim _{\omega \rightarrow 0} \kappa_{\omega}=\kappa_{0}=\left(\int_{0}^{1} e^{\psi} d z \int_{0}^{1} e^{-\psi} d z\right)^{-1}$.

(2) As $\omega \rightarrow \infty, \kappa_{\omega}$ converges to 1 from above, and as a consequence, $\omega \mapsto \kappa_{\omega}$ has a maximum, which is therefore always strictly bigger than 1 .

These properties are clearly observed in our two examples and we suspect that the first one is always true. 


\subsection{Rates of convergence}

Rates of convergence is a difficult issue. Using functional inequalities, we have proved in [2] that they can be estimated, but we suspect that much more could be done. Let us make the following conjectures:

(1) The rate of convergence is governed by the best constant in the logarithmic Sobolev inequality and not by the other terms in the expansion, which could anyway be controlled by a higher order computation.

(2) The best constant in the logarithmic Sobolev inequality is the same as for the other generalized Poincaré inequalities which interpolate between the logarithmic Sobolev inequality and the Poincaré inequality as already discussed in Section 2.

With these hypotheses, the sharp rate of convergence measured in $L^{1}$ norm is $1 / \tau$ (or $t^{-1 / \tau}$ in the original variables), with $\tau=2$ for $\omega=0$ and $\tau=2 \kappa_{\omega} / \kappa_{0}$ if $\omega>0$. As soon as we know that $\kappa_{\omega}>\kappa_{0}$, we already know from [2] (also see the discussion at the end of Section 3 and Section 5.4) that the above rate is an upper bound.

Numerically, understanding the rate of convergence is an extremely difficult question, corresponding to a stiff problem, with no well adapted basis, as the oscillating (small scale) variable depends on $t$.

\section{Conclusion}

Our results are based on a very simple model, but show how to compute analytically and numerically various quantities which are not easy to obtain by direct MonteCarlo simulations. The main difficulty comes from the oscillatory behavior of the potential, which is very clear in self-similar variables, and results in highly non-trivial attractors. Mathematically, this can be handled with the tools of homogenization theory, which provide an equation for the macroscopic profile and formulae for the two main parameters, the speed $c_{\omega}$ of the center of mass (or drift velocity) and the effective diffusion coefficient $\kappa_{\omega}$. This should not hide a major mathematical difficulty: the time $t$ is not independent of the small parameter in the homogenization approach, namely $1 / \sqrt{t}$, in the original variables. Moreover, several length scales have to be taken into account. The position of the center of mass is of the order of $t$, while the typical size of the front grows like $\sqrt{t}$. Typical relaxation rates are exponential at small scale, but of the order of $t^{-1 / \tau}$ or $1 / \sqrt{t}$ when measured globally. Hence asymptotic expansions are not at all easy to handle even at a formal level and quite hard to justify. The way out for such difficulties is the homogenized logarithmic Sobolev inequality, with its own difficulties. The inequality anyway has the very nice feature of connecting the rates of convergence with $\kappa_{\omega}$, something which definitely should be further investigated from a numerical point of view. 
However, knowing $c_{\omega}$ and $\kappa_{\omega}$ accurately is a major step in the understanding of the asymptotic behavior of the solutions of the stochastic Stokes' drift. It gives solid grounds to a notion of effective diffusion. A striking consequence is that it gives a new criterion for measuring the efficiency of coherent transport using the number $\mathrm{E}$.

We hope that our contribution will contribute to more realistic models from a theoretical point of view and will be used for benchmarking the numerous simulations that are being performed mostly with Monte-Carlo approaches.

Acknowledgments. A.B. and J.D. have been partially supported ECOS-CONICYT \# C05E09. J.D. wishes to thank the members of the DIM for their hospitality. A.B. acknowledges the support of the KAUST investigator award. M.K. has been partially supported by: FONDECYT 1050311, Nucleo Milenio P04-069-F, FONDAP and ECOS-CONICYT \# C05E05. All computations have been performed with Mathematica ${ }^{\mathrm{TM}}$.

(C) 2008 by the authors. This paper may be reproduced, in its entirety, for non-commercial purposes.

\section{References}

[1] I. Bena, C. Van den Broeck, and M. Copelli, J. Stat. Phys. 101, 415 (2000).

[2] A. Blanchet, J. Dolbeault, and M. Kowalczyk, Stochastic Stokes' drift, homogenized functional inequalities, and large time behaviour of Brownian ratchets, Preprint, 2008.

[3] A. J. Majda and P. R. Kramer, Phys. Rep. 314, 237 (1999).

[4] T. Goudon and F. Poupaud, Comm. Partial Differential Equations 26, 537 (2001).

[5] M. Vergassola and M. Avellaneda, Phys. D 106, 148 (1997).

[6] T. Goudon and F. Poupaud, SIAM J. Math. Anal. 36, 856 (2004/05).

[7] K. M. Jansons and G. D. Lythe, Phys. Rev. Lett. 81, 3136 (1998).

[8] M. O. Magnasco, Phys. Rev. Lett. 71, 1477 (1993).

[9] C. V. den Broeck, Europhysics Letters 46, 1 (1999).

[10] M. Borromeo and F. Marchesoni, Phys. Lett. A 249, 199 (1998).

[11] P. Hänggi, F. Marchesoni, and F. Nori, Ann. Phys. (8) 14, 51 (2005).

[12] P. Reimann, Phys. Rep. 361, 57 (2002).

[13] P. Reimann and P. Hänggi, Applied Physics A: Materials Science \& Processing 75, 169 (2002).

[14] D. Reguera et al., Physical Review Letters 96, 130603 (2006).

[15] A. Ajdari and J. Prost, C. R. Acad. Sci. Paris, Série II 315, 1653 (1992).

[16] F. Jülicher, A. Ajdari, and J. Prost, Rev. Mod. Phys. 69, 1269 (1997).

[17] A. Parmeggiani, F. Jülicher, A. Ajdari, and J. Prost, Phys. Rev. E 60, 2127 (1999). 
[18] P. Reimann et al., Phys. Rev. Lett. 87, 010602 (2001).

[19] P. Reimann et al., Phys. Rev. E 65, 031104 (2002).

[20] G. Costantini and F. Marchesoni, Europhysics Letters 48, 491 (1999).

[21] H. Sakaguchi, Journal of the Physical Society of Japan 75, 124006 (2006).

[22] K. Sasaki and S. Amari, Journal of the Physical Society of Japan 74, 2226 (2005).

[23] K. Hayashi and S.-i. Sasa, Phys. Rev. E 69, 066119 (2004).

[24] T. Harada and K. Yoshikawa, Phys. Rev. E 69, 031113 (2004).

[25] L. P. Faucheux, G. Stolovitzky, and A. Libchaber, Phys. Rev. E 51, 5239 (1995).

[26] S. Lifson and J. L. Jackson, The Journal of Chemical Physics 36, 2410 (1962).

[27] S. Lee and D. Grier, Physical Review Letters 96, 190601 (2006).

[28] V. Blickle, T. Speck, U. Seifert, and C. Bechinger, Physical Review E 75, 60101 (2007).

[29] M. Evstigneev et al., Phys Rev E 77, 041107 (2008).

[30] J. Regtmeier, T. Duong, R. Eichhorn, D. Anselmetti, and A. Ros, Analytical Chemistry 79, 3925 (2007).

[31] R. D. Astumian, Science 276, 917 (1997).

[32] M. Bier, Physics Letters A 211, 12 (1996).

[33] C. R. Doering, W. Horsthemke, and J. Riordan, Phys. Rev. Lett. 72, 2984 (1994).

[34] P. Reimann, Physics Reports 290, 149 (1997).

[35] M. Kostur, L. Machura, P. Hanggi, J. Luczka, and P. Talkner, Physica A: Statistical Mechanics and its Applications 371, 20 (2006).

[36] R. Bartussek, P. Hanggi, and J. Kissner, Europhys. Lett 28, 459 (1994).

[37] J. Kula, T. Czernik, and J. Luczka, Physics Letters A 214, 14 (1996).

[38] M. Kostur, G. Knapczyk, and J. Luczka, Physica A: Statistical Mechanics and its Applications 325, 69 (2003).

[39] J. Luczka, R. Bartussek, and P. Hanggi, Europhys. Lett 31, 431 (1995).

[40] H.-Y. Wang and J.-D. Bao, Physica A: Statistical Mechanics and its Applications 374, 33 (2007).

[41] J. A. Freund and L. Schimansky-Geier, Phys. Rev. E 60, 1304 (1999). 